\title{
Neurological manifestations of COVID-19: a review of what we know so far
}

\author{
Panagiotis Gklinos ${ }^{1}$ (D)
}

Received: 22 April 2020 / Revised: 17 May 2020 / Accepted: 19 May 2020 / Published online: 26 May 2020

c) Springer-Verlag GmbH Germany, part of Springer Nature 2020

\begin{abstract}
Coronavirus disease 2019 (COVID-19) has become a pandemic disease globally. While it mostly presents with respiratory symptoms, it has already been found that it could manifest with a series of neurological symptoms as well, either at presentation or during the course of the disease. Symptoms vary from non-specific such as headache or dizziness to more specific such as convulsions and cerebrovascular disease (CVD). This study aims to give an overview of the neurological manifestations of COVID-19 and discuss the potential pathogenetic mechanisms of central nervous system (CNS) involvement. Clinicians and especially internists, neurologists, and infectious disease specialists should be aware of these symptoms and able to recognize them early. Prompt diagnosis and immediate management of the neurological manifestations of the novel coronavirus will not only improve the prognosis of COVID-19 patients but will also prevent the dissemination of the disease due to misdiagnosed cases.
\end{abstract}

Keywords COVID-19 $\cdot$ Coronavirus $\cdot$ SARS-CoV-2 $\cdot$ Neurological manifestations $\cdot$ Neurology

\section{Introduction}

Coronavirus Disease 2019 (COVID-19) epidemic emerged in December 2019 in Wuhan, China and has rapidly spread to the rest of the world. The outbreak has been declared a world pandemic by WHO on 11 March 2020, with more than 2,500,000 cases worldwide so far [1]. COVID-19 is confirmed to be caused by a novel coronavirus (2019 novel coronavirus, 2019-nCoV) and presents with symptoms similar to those of severe acute respiratory syndrome coronavirus (SARS-CoV) in 2003. Both viruses shared the same receptor, angiotensin-converting enzyme 2 (ACE2) [2], thus, the novel virus was named SARS-CoV-2.

COVID-19 typically presents with fever and respiratory symptoms including dry cough and dyspnea. The elderly population is more likely to develop severe disease complications, especially those with comorbidities such as hypertension, diabetes mellitus, and chronic obstructive pulmonary disease (COPD). However, neurological manifestations of the novel coronavirus are not precepted by all clinicians,

Panagiotis Gklinos

gklinosp@gmail.com

1 Neurology Department, KAT General Hospital of Attica, 2 Nikis Str., Kifisia, Attica, 14561 Athens, Greece thus, leading to inappropriate management of COVID-19 patients presenting with non-specific neurological symptoms initially. This article aims to review the cases, which reported neurological symptoms at presentation or during the course of the disease and discuss the potential mechanisms of Central Nervous System (CNS) involvement in COVID-19.

\section{SARS-CoV-2 overview}

Coronaviruses (CoVs) have an average diameter of $100 \mathrm{~nm}$, and they are spherical or oval. They are enveloped viruses with a single-strand, positive sense RNA-genome. The SARS-related coronaviruses are covered by spike proteins and when observed in the electron microscopy they have a typical crown-like shape. Spike proteins contain a variable receptor-binding domain (RBD), which binds to angiotensinconverting enzyme-2 (ACE-2) receptor found in the heart, lungs, kidneys, and gastrointestinal tract, thus, facilitating viral entry into target cells [2, 4].

SARS-CoV-2, along with SARS-CoV and Middle East respiratory syndrome coronavirus (MERS-CoV), is among the seven known coronaviruses that can infect humans with a genome length of 29,000 nucleotide bases, which preserve 
the genetic information for its reproduction. The genetic similarity between SARS-CoV-2 and bat coronavirus is $96 \%$ whilst its genetic similarity with SARS-CoV is $79.5 \%$ [4]. Therefore, it is believed that the novel coronavirus originated from bats and after mutating, it infected other animals and humans. Malayan pangolin is considered the intermediate host of SARS-CoV-2 [5].

SARS-CoV-2 is thought to be transmitted most readily by respiratory droplets. Moreover, the digestive tract might be another transmission route as the virus has been detected in human stool [6]. Additionally, it is believed that it can be transmitted through aerosols under prolonged exposure, in a closed environment.

\section{Neurological manifestations of COVID-19}

\section{Encephalitis-meningitis}

Two cases of Sars-CoV-2-related encephalitis have been reported so far [7, 8]. The first one, is a 24-year-old man in Yamanashi, Japan, who initially complained about headaches, fever, and fatigue. He was prescribed antipyretic agents and laninamivir under the diagnosis of influenza and was advised to stay home. However, a few days later he was found unconscious and was transferred to the hospital, where he presented with generalized seizures, Glasgow coma scale (GCS) of 6 and neck stiffness. Brain magnetic resonance imaging (MRI) showed hyperintensity along the wall of the inferior horn of the right lateral ventricle and hyperintense signal changes in the right mesial temporal lobe and hippocampus with slight hippocampal atrophy in diffusion-weighted images (DWI) and fluid-attenuated inversion recovery images (FLAIR), indicating right lateral ventriculitis and encephalitis mainly on right mesial lobe and hippocampus. Although the specific SARS- CoV-2 RNA was not detected in the nasopharyngeal swab, it was detected in CSF confirming the diagnosis of Meningitis/Encephalitis associated with SARS-Coronavirus-2. The other case was reported in Ditan Hospital, Beijing, China, where a 56-yearold patient diagnosed with COVID-19 developed an altered level of consciousness. Although he had normal brain computerized tomography (CT) scans, cerebrospinal fluid (CSF) testing for SARS-CoV-2 was positive, confirming the diagnosis of encephalitis due to the novel coronavirus.

\section{Encephalopathy}

The first case was reported in Detroit, Miami, and is about a female airline worker in her late fifties presented with a 3-day history of cough, fever, and altered mental status [9]. She was diagnosed with COVID-19 through the detection of the RNA of the novel coronavirus in the nasopharyngeal swab. However, due to a traumatic lumbar puncture, CSF testing for SARS-CoV-2 was unable to be performed. Noncontrast brain CT scan demonstrated symmetric hypoattenuation within the bilateral medial thalami. Brain MRI demonstrated hemorrhagic rim enhancing lesions within the bilateral thalami, medial temporal lobes, and subinsular regions. Clinical presentation and imaging findings were consistent with the diagnosis of acute necrotizing encephalopathy (ANE), a rare complication of influenza and other viral infections, which has been related to intracranial cytokine storms, resulting in blood-brain-barrier breakdown [10]. The patient was treated with intravenous immunoglobulin. The second case concerns a 74-yearold patient, presented at the emergency department (ED) with fever and cough, who was initially discharged home with oral antibiotics [11]. He returned to the ED within $24 \mathrm{~h}$ with worsening symptoms, including headache, altered mental status, fever, and cough. Upon examination, the patient was found encephalopathic, nonverbal, and unable to follow any commands. Non-contrast, brain CT scan showed no acute abnormalities whilst electroencephalography (EEG) showed bilateral slowing and focal slowing in the left temporal region with sharply countered waves. The patient was found to be positive for COVID-19 (nasopharyngeal swab), whilst CSF testing did not reveal a central nervous system infection.

\section{Cerebrovascular disease}

Two studies have been conducted so far, regarding the association of COVID-19 and cerebrovascular disease (CVD). The first one is a retrospective, observational analysis of consecutive COVID-19 patients admitted to Union Hospital, Wuhan, China [12]. It showed that CVD is not uncommon during SARS-CoV-2 infection, especially in older patients with risk factors. More specifically, 5\% of the patients developed acute ischemic stroke, $0.5 \%$ cerebral venous sinus thrombosis, and $0.5 \%$ cerebral hemorrhage. Patients who developed CVD were more likely to present with severe COVID-19, and to have cardiovascular risk factors such as hypertension, diabetes, and previous medical history of CVD. The other study is a retrospective case series in Wuhan, China, which reported the neurological symptoms of COVID-19 patients [13]. It showed that patients with severe COVID-19 were more likely to develop CVD as compared with non-severe cases. (5.7\%four cases of acute ischemic stroke and one case of cerebral hemorrhage vs. $0.8 \%$-one patient with ischemic stroke). However, we should mention that both studies were conducted in Wuhan, China, thus, findings cannot be applied to the general population. 


\section{Olfactory and gustatory disorders}

It is well-known that viral infections including coronaviruses can lead to smell dysfunction $[14,15]$. Post-viral anosmia is one of the leading causes of loss of sense of smell in adults, accounting for up to $40 \%$ cases of anosmia [16]. The underlying cause is primarily mucosal congestion, which leads to nasal obstruction and conductive olfactory loss [16]. However, the novel coronavirus is thought to cause olfactory and gustatory disorders without causing rhinorrhea or nasal obstruction. A multi-center European study showed that $85.6 \%$ and $88.8 \%$ of COVID-19 patients developed olfactory and gustatory disorders respectively [17]. Regarding the olfactory disorders, $79.6 \%$ of the patients were anosmic and $20.4 \%$ were hyposmic. Interestingly, among the $18.2 \%$ of patients without nasal obstruction or rhinorrhea, $79.7 \%$ were hyposmic or anosmic, suggesting that the inflammatory reaction of the nasal mucosa is not the cause of smell dysfunction in this case. The gustatory dysfunction consisted of reduced, discontinued, or distorted ability to taste flavors (salty, sweet, bitter, and sour) in $78.9 \%$ and $21.1 \%$ of patients, respectively.

\section{Other neurological symptoms}

Besides the clinical manifestations mentioned before, SARS$\mathrm{CoV}-2$ can potentially present with a number of non-specific neurological symptoms. The above-mentioned clinical case series in Wuhan, China, showed that $36.4 \%$ of COVID-19 patients had neurological symptoms at onset. The most common neurological symptoms were dizziness (16.8\%), headache $(13.1 \%)$, skeletal muscle inflammation $(10.7 \%)$, and altered mental status including confusion, disorientation, and impaired level of consciousness (14.8\%) [13]. Nervous system symptoms were significantly more frequent in patients with severe COVID-19 as compared to non-severe cases $(45.5 \%)$ vs. (30.2\%). The severity of COVID-19 was defined by the international guidelines for communityacquired pneumonia [18].

\section{Guillain-Barré syndrome}

Finally, a case of Guillain-Barré syndrome should be discussed, even though it only suggests a possible association with SARS-CoV-2 and not a proven causal relationship [19]. A 61-year-old woman without respiratory symptoms presented to the hospital with acute weakness in both legs and severe fatigue, progressing within one day. Upon examination she disclosed symmetric weakness (4/5) and areflexia in both legs and feet. Three days after admission, her symptoms progressed. Muscle strength was grade $4 / 5$ in both arms and hands and 3/5 in both legs and feet. Sensation to light touch and pinprick was decreased distally. CSF testing showed increased protein levels and nerve conduction studies showed delayed distal latencies and absent $\mathrm{F}$ waves in early course, supporting demyelinating neuropathy. The woman was diagnosed with Guillain-Barré syndrome. Seven days after admission she developed dry cough, fever $\left(38.2{ }^{\circ} \mathrm{C}\right)$ and she was found to be positive for COVID-19 (nasopharyngeal swab). Although she had not initially presented with respiratory symptoms, it has been shown that sometimes SARS-CoV-2 may manifest in a non-specific way (fever in only 43.8\%). Moreover, the patient's initial laboratory abnormalities (lymphocytopenia and thrombocytopenia), were consistent with the clinical characteristics of patients with COVID-19. Unfortunately, the absence of coronavirus testing on admission does not allow us to state a causal relationship between the novel coronavirus and Guillain-Barré syndrome. However, it certainly suggests that a parainfectious pattern of the syndrome may exist and neurologists should be aware of the potential association.

\section{Discussion}

As the new epidemic is still ongoing, documentation of the neurological manifestations of SARS-CoV-2 is scarce. Patients with COVID-19 may initially present with nonspecific neurological symptoms including headache and dizziness. Others can develop more specific symptoms such as seizures and CVD. It has also been shown, that the more severe the infection, the more likely it is to develop neurological symptoms, especially CVD and altered mental status [13].

Human coronaviruses have already been found to be neuroinvasive and neurotropic [20, 21]. More specifically, SARS-CoV has been shown to induce various neurological diseases such as polyneuropathy, encephalitis, and aortic ischemic stroke [22]. In addition, its RNA has been detected in the CSF of a patient with the severe acute respiratory syndrome (SARS) while autopsy samples from eight patients with SARS revealed the presence of SARS-CoV in brain samples by immunohistochemistry, electron microscopy, and real-time-PCR (RT-PCR) [23, 24]. MERS-CoV is also a neuroinvasive coronavirus, which has been linked to a series of neurological manifestations including altered mental status, ischemic stroke, and Guillain-Barré syndrome [25]. The genetic and structural similarities of SARS-CoV-2 with SARS-CoV and MERS-CoV, indicate that the novel coronavirus could potentially invade CNS using the same pathophysiological mechanisms the other coronaviruses use. Although, the exact pathophysiological mechanisms are not fully understood two possible theories have been proposed so far: hematogenous dissemination and neuronal retrograde dissemination. The first one requires the presence of a given virus in the blood, where it can either infect the endothelial 
cells of the blood-brain-barrier (BBB), or infect leukocytes that will become some sort of viral reservoir for dissemination to other sites [26]. On the other hand, neuronal retrograde dissemination occurs when a virus migrates by infecting sensory or motor nerve endings, achieving retrograde or anterograde neuronal transport, by using the cell machinery of active transport to access the CNS [26, 27]. Olfactory nerves and the olfactory bulb in the nasal cavity may work as a connecting channel between the nasal cavity and the CNS [28]. The latter scenario is further supported by the fact that many patients with COVID-19 experience anosmia or hyposmia [18]. Moreover, removal of the olfactory bulb in the mice resulted in a restricted invasion of $\mathrm{CoV}$ into the CNS [29].

Regarding the occurrence of acute cerebrovascular events, patients with severe COVID-19 are found to have increased levels of serum D-dimers, thus are more likely to develop acute embolic vascular events [30]. Also, the cytokine storm syndrome that the novel coronavirus causes, may lead to CVD as well [31]. Finally, thrombocytopenia that critically ill patients present, as well as blood pressure fluctuations in hypertensive patients due to SARS-CoV-2 binding to the ACE2 receptor may increase the risk of cerebral hemorrhage [30].

\section{Conclusion}

Although the respiratory manifestations of SARS-CoV-2 are well recognized, the neurological manifestations have not been adequately studied yet. Critically ill patients are at a greater risk of developing neurological symptoms during the course of the disease. Physicians should be aware of these symptoms and evaluate patients early to avoid the dissemination of the disease and improve the prognosis of patients. Continuing documentation of neurological symptoms and signs, timely CSF testing, EEG, and brain autopsies of COVID-19 patients who did not survive may help us understand better the neurological manifestations of the novel coronavirus as well as the pathophysiological mechanisms of CNS involvement.

Funding This research received no specific grant from any funding agency in the public, commercial, or not-for-profit sectors.

\section{Compliance with ethical standards}

Conflicts of interest Dr. P. Gklinos reports no disclosures.

\section{References}

1. Mahase E (2020) Covid-19: WHO declares pandemic because of "larming levels" of spread, severity, and inaction. BMJ 368:1036
2. Zhao Y, Zhao Z, Wang Y et al (2020) Single-cell RNA expression profiling of ACE2, the putative receptor of Wuhan 2019nCov. BioRxiv. https://doi.org/10.1101/2020.01.26.919985

3. Ksiazek TG, Erdman D, Goldsmith CS, Zaki SR, Peret T, Emery S, Tong S, Urbani C, Comer JA, Lim W et al (2003) A novel coronavirus associated with severe acute respiratory syndrome. N Engl J Med 348:1953-1966

4. Wu A, Peng Y, Huang B, Ding X, Wang X, Niu P et al (2020) Genome composition and divergence of the novel coronavirus (2019-nCoV) originating in China. Cell Host Microbe 27(3):325-328

5. Cyranoski D (2020) Did pangolins spread the China coronavirus to people? Nature 2020.

6. Yong Z, Cao C, Shuangli Z et al (2020) Notes from the field: isolation of 2019-nCoV from a stool specimen of a Laboratoryconfirmed case of the coronavirus disease 2019 (COVID-19). China CDC Weekly 2:123-124

7. Moriguchi T, Harii N, Goto J, Harada D, Sugawara H, Takamino J, Ueno M, Sakata H, Kondo K, Myose N, Nakao A, Takeda M, Haro H, Inoue O, Suzuki-Inoue K, Kubokawa K, Ogihara S, Sasaki T, Kinouchi H, Kojin H, Ito M, Onishi H, Shimizu T, Sasaki Y, Enomoto N, Ishihara H, Furuya S, Yamamoto T, Shimada S (2020) A first case of Meningitis/Encephalitis associated with SARS-Coronavirus-2. Int J Infect Dis. https://doi. org/10.1016/j.ijid.2020.03.062

8. Xiang P, Xu XM, Gao LL, Wang HZ, Xiong HF, Li RH et al (2020) First case of 2019 novel coronavirus disease with encephalitis. ChinaXiv 202003:00015

9. Poyiadji N, Shahin G, Noujaim D, Stone M, Patel S (2020) Brent Griffith; COVID-19-associated acute hemorrhagic necrotizing encephalopathy: CT and MRI. Radiology 31:201187. https:// doi.org/10.1148/radiol.2020201187

10. Rossi A (2008) Imaging of acute disseminated encephalomyelitis. Neuroimaging Clin 18(1):149-161

11. Filatov A, Sharma P, Hindi F et al (2020) Neurological complications of coronavirus disease (COVID-19): encephalopathy. Cureus 12(3):e7352. https://doi.org/10.7759/cureus.7352

12. Li Y, Wang M, Zhou Y, Chang J, Xian Y, Mao L, Hong C, Chen S, Wang Y, Wang H, Li M, Jin H, Hu B (2020) Acute cerebrovascular disease following COVID-19: a single center, retrospective, observational study (3/3/2020). Doi: 10.2139/ ssrn. 3550025

13. Mao L, Wang M, Chen S, He Q, Chang J, Hong C, Zhou Y, Wang D, Li Y, Jin H, Hu B (2020) Neurological manifestations of hospitalized patients with COVID-19 in Wuhan, China: a retrospective case series study. MedRxiv 2020.02.22.20026500. Doi: 10.1101/2020.02.22.20026500

14. Suzuki M, Saito K, Min WP, Vladau C, Toida K, Itoh H, Murakami S (2007) Identification of viruses in patients with postviral olfactory dysfunction. Laryngoscope 117(2):272-277

15. Van Riel D, Verdijk R, Kuiken T (2015) The olfactory nerve: a shortcut for influenza and other viral diseases into the central nervous system. J Pathol 235(2):277-287. https://doi.org/10.1002/ path.4461

16. Welge-Lussen A, Wolfensberger M (2006) Olfactory disorders following upper respiratory tract infections. Taste and smell, 63. Karger Publishers 2006:125-132

17. Lechien JR, Chiesa-Estomba CM, De Siati DR et al (2020) Olfactory and gustatory dysfunctions as a clinical presentation of mildto-moderate forms of the coronavirus disease (COVID-19): a multicenter European study. Eur Arch Otorhinolaryngol. https://doi. org/10.1007/s00405-020-05965-1

18. Zhao H, Shen D, Zhou H, Liu J, Chen S (2020) Guillain-Barré syndrome associated with SARS-CoV-2 infection: causality or coincidence? Lancet Neurol. pii: S1474-4422(20)30109-5. doi: 10.1016/S1474-4422(20)30109-5. 
19. Metlay JP, Waterer GW, Long AC et al (2019) Diagnosis and treatment of adults with community-acquired pneumonia: an official clinical practice guideline of the American Thoracic Society and Infectious Disease Society of America. Am J Respir Crit Care Med 200:e45-e67

20. Desforges M, Le Coupanec A, Dubeau P et al (2020) Human coronaviruses and other respiratory viruses: underestimated opportunistic pathogens of the central nervous system? Viruses 12:14

21. Desforges M, Le Coupanec A, Brison E et al (2014) Neuroinvasive and neurotropic human respiratory coronaviruses: potential neurovirulent agents in humans. Adv Exp Med Biol 807:75-96

22. Tsai LK, Hsieh ST, Chang YC (2005) Neurological manifestations in severe acute respiratory syndrome. Acta Neurol Taiwan 14(3):113-119

23. Hung EC, Chim SS, Chan PK et al (2003) Detection of SARS coronavirus RNA in the cerebrospinal fluid of a patient with severe acute respiratory syndrome. Clin Chem 49:2108-2109

24. Gu J, Gong E, Zhang B et al (2005) Multiple organ infection and the pathogenesis of SARS. J Exp Med 202(3):415-424

25. Kim JE, Heo JH, Kim HO, Song SH, Park SS, Park TH et al (2017) Neurological complications during treatment of middle east respiratory syndrome. J Clin Neurol 13(3):227-233
26. Berth SH, Leopold PL, Morfini GN (2009) Virus-induced neuronal dysfunction and degeneration. Front Biosci 14:5239-5259

27. Swanson PA, McGavern DB (2015) Viral diseases of the central nervous system. Curr Opin Virol 11:44-54

28. Koyuncu OO, Hogue IB, Enquist LW (2013) Virus infections in the nervous system. Cell Host Microbe 13(4):379-393. https:// doi.org/10.1016/j.chom.2013.03.010

29. Bohmwald K, Galvez NMS, Rios M, Kalergis AM (2018) Neurologic alterations due to respiratory virus infections. Front Cell Neurosci 12:386

30. Wang Y, Wang Y, Chen Y, Qin Q (2020) Unique epidemiological and clinical features of the emerging 2019 novel coronavirus pneumonia (COVID-19) implicate special control measures. J Med Virol. 92(6):568-576

31. Mehta P, McAuley DF, Brown M, Sanchez E, Tattersall RS, Manson JJ (2020) COVID-19: consider cytokine storm syndromes and immunosuppression. Lancet 395(10229):1033-1034 